Eur J Clin Chem Clin Biochem

1995; 33:503-512

(c) 1995 Walter de Gruyter \& Co.

Berlin - New York

\title{
Influence of Photoisomers in Bilirubin Determinations on Kodak Ektachem and Hitachi Analysers in Neonatal Specimens Study of the Contribution of Structural and Configurational Isomers
}

\author{
By Jean-Marc Gulian ${ }^{1,2}$, Christiane Dalmasso ${ }^{1,2}$, Véronique Millet ${ }^{3}$, Daniel Unal ${ }^{3}$ and Michel Charrel ${ }^{1,2}$ \\ ${ }^{1}$ Laboratoire de Chimie Biologique, Faculté de Médecine, Marseille, France \\ 2 Service de Biochimie, CHU Timone, Marseille, France \\ 3 Service de Médecine Infantile, CHU Timone, Marseille, France
}

(Received November 28, 1994/April 19, 1995)

Summary: We compared data obtained with the Kodak Ektachem and Hitachi 717 Analysers and HPLC from 83 neonates under phototherapy. Total bilirubin values determined with the Kodak and Hitachi are in good agreement, but we observed a large discrepancy in the results for conjugated (Kodak) and direct (Hitachi) bilirubin. HPLC revealed that all the samples contained configurational isomers, while only $7.7 \%$ and $30.8 \%$ contained conjugated bilirubin and structural isomers, respectively. We developed a device for the specific and quantitative production of configurational or structural isomers, by irradiation with blue or green light. In vitro, total bilirubin values are coherent for the routine analysers in the presence of configurational or structural isomers. With configurational isomers, unconjugated bilirubin (Kodak) is lower than total bilirubin (Kodak), and conjugated bilirubin (Kodak) is always equal to zero, so the apparatus gives a false positive response for delta bilirubin. In contrast, the direct bilirubin (Hitachi) is constant. Furthermore, in the presence of structural isomers, unconjugated bilirubin (Kodak) is unexpectedly higher than total bilirubin (Kodak), conjugated bilirubin (Kodak) is proportional to the quantity of these isomers, and direct bilirubin (Hitachi) is constant. The contribution of photoisomers in bilirubin measurements is discussed.

\section{Introduction}

In jaundiced newborns, conventional phototherapy is required for lowering hyperbilirubinaemia and sometimes, in severe jaundice, intensive phototherapy with a high light energy level. This intensive phototherapy, which is as efficient as exchange transfusion, induces a rapid decrease of unconjugated bilirubin, of about $34-45 \%$ after 4 hours (1). The lowering of plasma unconjugated bilirubin during phototherapy is due to the appearance of new molecular species by 3 pathways: photooxidation, and structural and configurational isomerization of the native form $(4 Z, 15 Z)$-bilirubin IX $\alpha$ (2). These photoproducts are more polar than the native form and can be excreted in urine and bile without conjugation. In vitro, photoisomerization appears as the initial process (3), followed by formation of biliverdin (4), then photo- oxidation and the appearance of colourless photodegradation compounds (5). The mechanism of bilirubin photooxidation has been reviewed by Landen et al. (3), and the resulting compounds were first identified by Lightner et al. (6) in the urine of jaundiced newborns under phototherapy. Nevertheless, this process is too slow in vivo to account for the rapid decrease of bilirubin. Elimination is due principally to formation of photoisomers: configurational isomers $(4 E, 15 Z)-,(4 Z, 15 E)-,(4 E, 15 E)$ bilirubin IX $\alpha$, and mainly structural isomers $(4 E, 15 Z)$ cyclobilirubin IX $\alpha$ and $(4 E, 15 E)$-cyclobilirubin IX $\alpha$ (7) (fig. 1). Structural isomers are formed by irradiation with green lamps, whereas blue and white lamps produce configurational isomers $(8-10)$. The clinical efficiency of the different lamps and the effective doses are 


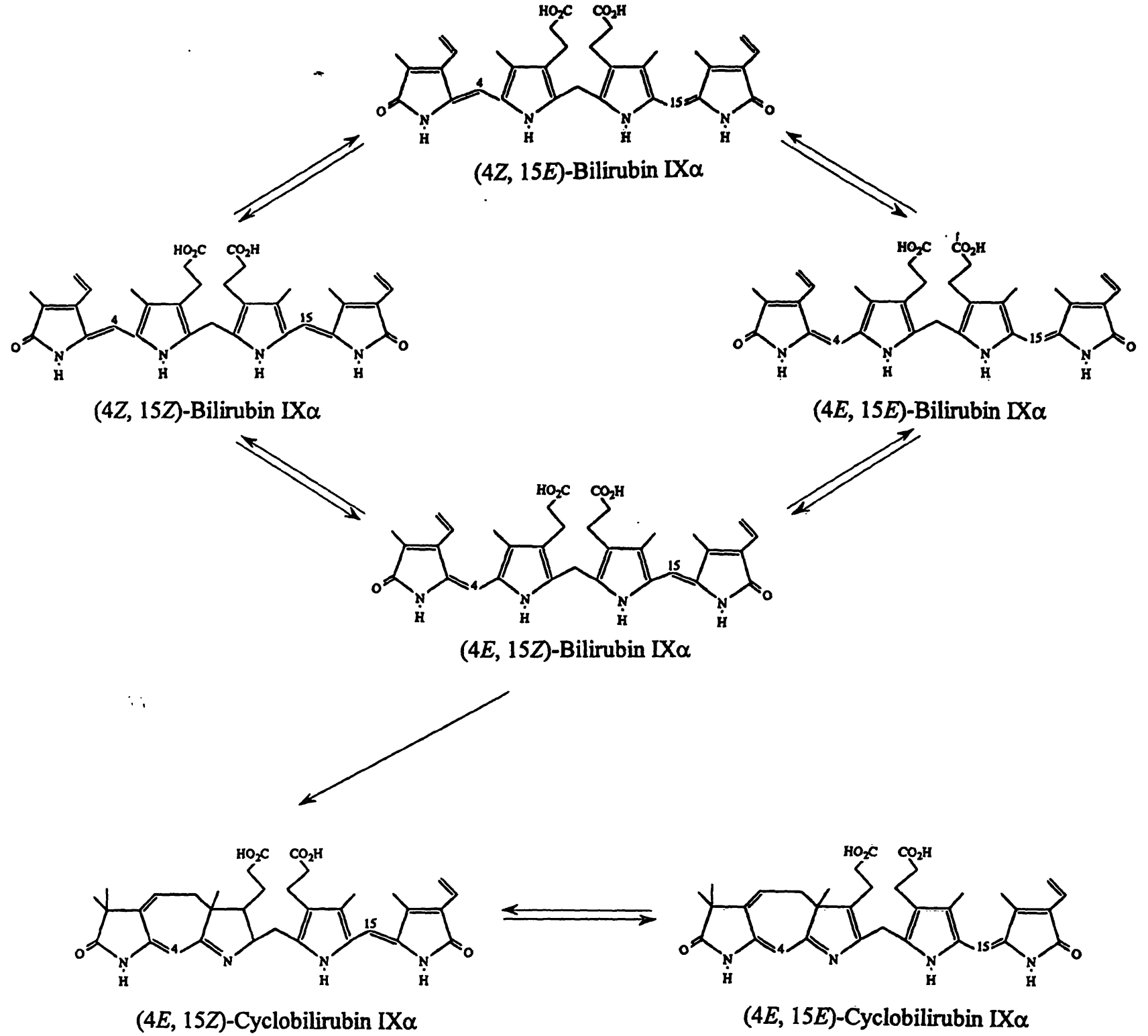

Fig. 1 Pathways of formation and structural formulae of the different bilirubin isomers.

controversial $(7,8,11-16)$. During phototherapy, frequent plasma bilirubin evaluations are required in order to follow the decrease of unconjugated bilirubin. Often, in samples from patients with high levels of unconjugated bilirubinaemia, we have observed differences in values for bilirubin fractions between the automated analysers used routinely in our laboratory. These discrepancies are found in the determination of conjugated bilirubin (CB), direct bilirubin (DB), total bilirubin (TB) and unconjugated bilirubin (UB), and they are particularly serious, because the analytical fractionation of bilirubin into direct- and indirect-fractions unfortunately does not correspond precisely to the pigments present in the serum of patients. A number of publications have previously highlighted these points $(17-24)$.

The purposes of this study were to investigate the influence of bilirubin photoisomers on the automated sys- tems currently used in laboratories for plasma bilirubin determinations.

\section{Materials and Methods}

\section{Phototherapy}

Standard phototherapy for icteric neonates was administered using 8 special blue lamps (Philips LL 20W/52) with $1-2 \mathrm{~mW} / \mathrm{cm}^{2}$ intensity in the 410 to $540 \mathrm{~nm}$ range. The exposure periods were variable, depending on biological results. Intensive multiple-directional phototherapy, with much higher intensity $\left(3.5-4 \mathrm{~mW} / \mathrm{cm}^{2}\right)$, was provided by an apparatus (Mediprema, France) equipped with 16 Philips TL 20W/52 lamps, 8 above and 8 below and given during a 4 hour period. Light intensities were measured by a radiometer VQ 460 (Verre and Quartz, Paris, France).

Sample collection

Arterial or venous blood samples from 83 newborns, collected after phototherapy, were centrifuged immediately. Plasma, protected 
from light, was analysed for bilirubin by routine tests. For HPLC analysis, samples were stored in the dark for a maximum of 2 weeks at $-20^{\circ} \mathrm{C}$. All the analyses for this study were performed on excess of blood samples from the clinical survey. Thus, sometimes, we did not have enough material to apply all the methodologies for all the samples.

\section{In vitro irradiation}

All experiments in vitro were conducted in a room under dimmed light. Solutions containing different concentrations of bilirubin were prepared from pooled plasma supplemented with a highly concentrated solution of bilirubin (crystalline bilirubin, Sigma, dissolved in $0.1 \mathrm{~mol} / 1 \mathrm{NaOH}$ ), containing a trace of EDTA (25). These solutions were gassed for $5 \mathrm{~min}$ with $99.9 \% \mathrm{~N}_{2}$. Irradiations were performed with a home-made device equipped with a $200-\mathrm{W}$ highpressure mercury lamp; wavelengths are selected by suitable blue filter (absorbance $>2$ for $\lambda<408 \mathrm{~nm}$ and $\lambda>417 \mathrm{~nm}$; minimal absorbance 0.38 for $\lambda=420-422 \mathrm{~nm}$ ) and green filter (absorbance $>2$ for $\lambda<426 \mathrm{~nm}$; minimal absorbance 0.17 for $\lambda$ between 530 to $556 \mathrm{~nm}$ ). Light intensities were $17-18 \mathrm{~mW} / \mathrm{cm}^{2}$ for the blue filter and $10-12 \mathrm{~mW} / \mathrm{cm}^{2}$ for the green filter. Plastic cuvettes (10 mm-pathlength) were filled with $0.8 \mathrm{ml}$ of bilirubin solutions, gassed with $99.9 \% \mathrm{~N}_{2}$, and irradiated. After irradiation, the solutions were kept at $-180^{\circ} \mathrm{C}$ in liquid nitrogen until analysis.

\section{Bilirubin routine analysis}

Bilirubin routine analysis was performed on a Kodak Ektachem 700 XR Analyser (Eastman Kodak Co. Rochester-Ny, USA) using multilayered slide methodology, and on a Hitachi 717 Automated Analyser (Hitachi, Tokyo, Japan). On the Hitachi, total bilirubin $_{\mathrm{Hitachi}}\left(\mathrm{TB}_{\mathrm{Hi}}\right)$ and direct bilirubin $(\mathrm{DB})$ were determined (26) and indirect bilirubin (indB) was calculated according to the relation: [indB $=\mathrm{TB}_{\mathrm{Hi}}-\mathrm{DB}$ ]. On the Kodak, total bilirubin Kodak $\left(\mathrm{TB}_{\mathrm{K}_{\mathrm{O}}}\right)$ was measured by a modified diazo method; unconjugated (UB) and conjugated bilirubin (CB) were determined by reflectance spectroscopy; the other quantities were calculated:

albumin-bound delta bilirubin $(\delta \mathrm{B})\left[\delta \mathrm{B}=\mathrm{TB}_{\mathrm{K}_{0}}-(\mathrm{UB}+\mathrm{CB})\right]$, direct bilirubin $\left(\mathrm{DB}_{\mathrm{K}_{0}}\right)\left[\mathrm{DB}_{\mathrm{Ko}_{\mathrm{o}}}=\mathrm{TB}_{\mathrm{Ko}_{\mathrm{o}}}-\mathrm{UB}=\mathrm{CB}+\delta \mathrm{B}\right]$ and neonatal bilirubin (NB) $[\mathrm{NB}=\mathrm{UB}+\mathrm{CB}]$.

We used TB slides, notwithstanding the fact that Kodak Company recommends that TB slides should not be employed for samples from neonates before 15 days $(27,28)$. Otherwise, we followed the recommendations of the manufacturers for bilirubin determinations, maintenance and calibration.

\section{High performance liquid chromatography}

We used a Waters liquid chromatography (Waters Assoc., Milford, MA, USA) equipped with a U6K injector and a two-pumps $(6000 \mathrm{~A})$ system. Absorbance was recorded at $455 \mathrm{~nm}$ with a spectrophotometer (GM 770). A D-6000 model data station and a L3000 photo diode array detector were employed to calculate peak areas and for acquiring spectra during the run (Merck-Hitachi). Separation was achieved at room temperature with a $7 \mu \mathrm{m} \mathrm{RP}-18$ Lichrosorb column $(250 \times 4 \mathrm{~mm}$ I. D.) from Merck (Darmstadt, Germany) at a flow rate of $1.0 \mathrm{ml} / \mathrm{min}$ in a two-solvent system. Solvent A was $100 \%$ acetonitrile. Solvent B was prepared by adding $40 \mathrm{ml}$ of dimethyl sulphoxide to $60 \mathrm{ml}$ of $0.1 \mathrm{~mol} / \mathrm{l}$ sodium acetate buffer $\mathrm{pH}=4.9$. For analytical separations, the following conditions were used: a linear gradient of $80 \%$ B to $70 \%$ B in 2 min, maintaining the gradient at $70 \%$ for $4 \mathrm{~min}$, a linear gradient of $70 \%$ B to $20 \%$ B in $21 \mathrm{~min}$, and $20 \%$ B during $5 \mathrm{~min}$. Samples were deproteinised by adding 5 volumes of acetonitrile/dimethyl sulphoxide $(4+1$, by vol.), followed vortex-mixing for $30 \mathrm{~s}$; after centrifugation $(3 \mathrm{~min}$ at $3000 \mathrm{~g}), 50 \mu \mathrm{l}$ of the supernatant were injected into the column. Identification of the photoisomers was based on chromatographic retention times. Extinction coefficients of photoisomers have been reported by several authors $(2,29,30)$ but the values do not always agree. As we used different HPLC separation conditions, the detector response was not corrected for absorption differences of photoisomers at this wavelength, and the results are expressed as peak area.

\section{Statistics}

Data were expressed as means, SD and CV. The areas of the HPLC peaks are expressed in arbitrary units. Linear regressions were calculated by the least-square method.

\section{Results}

\section{Standardization between Kodak and HPLC}

Different bilirubin calibrators from Kodak (human serum supplemented with purified bilirubin) were analysed by the Kodak method and by HPLC. These calibrators contain the 3 forms: $(4 Z, 15 Z)$-bilirubin IX $\alpha$, (4Z,15Z)-bilirubin XIII $\alpha$ and $(4 Z, 15 Z)$-bilirubin III $\alpha$, so we expressed the data as the sum of these 3 forms, i. e. $(4 Z, 15 Z)$-bilirubin (III +IX +XIII) $\alpha$. The results in figure 2 show that the peak area of $(4 Z, 15 Z)$-bilirubin (III+IX+XIII) $\alpha$ and conjugated bilirubin (CB HPLC) (expressed as the sum of mono- and diconjugated) were highly correlated to unconjugated (UB) (fig. 2a) and conjugated bilirubin (CB) (fig. 2b), respectively, as determined by the Kodak method. Consequently it might be possible to calculate the concentrations of conjugated (CB) and unconjugated bilirubin (UB) from HPLC peaks areas using the following equations:

$$
\begin{aligned}
& \text { UB }(\mu \mathrm{mol} / \mathrm{l}) \\
& =0.0205[(4 Z, 15 Z) \text {-bilinubin (III + IX }+ \text { XIII }) \alpha \\
& \text { peak area }]+3.35 \\
& \mathrm{CB}(\mu \mathrm{mol} / \mathrm{l})=0.0719(\mathrm{CB} \text { HPLC peak area })-1.46
\end{aligned}
$$

\section{Comparison of Kodak, Hitachi and HPLC for samples from neonates}

Measurement of total bilirubin in samples from neonates with the Kodak $\left(\mathrm{TB}_{\mathrm{Ko}}\right)$ and the Hitachi $\left(\mathrm{TB}_{\mathrm{Hi}}\right)$ (fig. 3a) demonstrated the high correlation between the two analysers for this quantity. On the other hand, conjugated and direct bilirubin display a less significant correlation (fig. $3 b$ ); this result is not surprising in view of the different methodologies used by the two analysers for these quantities. Figure $3 \mathrm{c}$ shows the plots of unconjugated and indirect bilirubin versus $(4 Z, 15 Z)$-bilirubin IX $\alpha$ determined by HPLC. The two least-square regression lines are different for the two quantities, unconjugated and indirect bilirubin. In addition, the measured value for unconjugated bilirubin was slightly different from unconjugated bilirubin calculated from equation (1), and indirect bilirubin is in poor agreement with the calcu- 
lated unconjugated bilirubin (data not shown). The results of HPLC runs $(n=52)$ from individual neonates are reported in table 1 . The mean, median and range values of $(4 Z, 15 Z)$-bilirubin IX $\alpha$ and conjugated bilirubin are expressed as peak area and in $\mu \mathrm{mol} / \mathrm{l}$ calculated from equations (1) and (2). For the photoisomers, we expressed the results only by peak area. The HPLC chromatograms revealed that blood from a large number $(98 \%)$ of patients contained a non-negligible amount of configurational isomers, while $7.7 \%$ contained conjugated bilirubin and $38.8 \%$ contained structural isomers.

\section{In vitro experiments}

For all in vitro studies, we performed experiments on samples of pooled plasma, without conjugated bilirubin, supplemented with bilirubin. Samples were irradi-

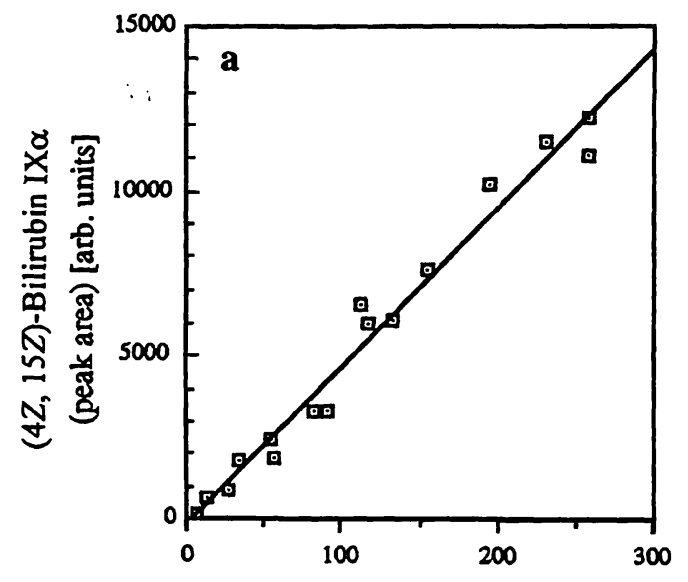

Unconjugated bilirubin [ $\mu \mathrm{mol} / 1]$

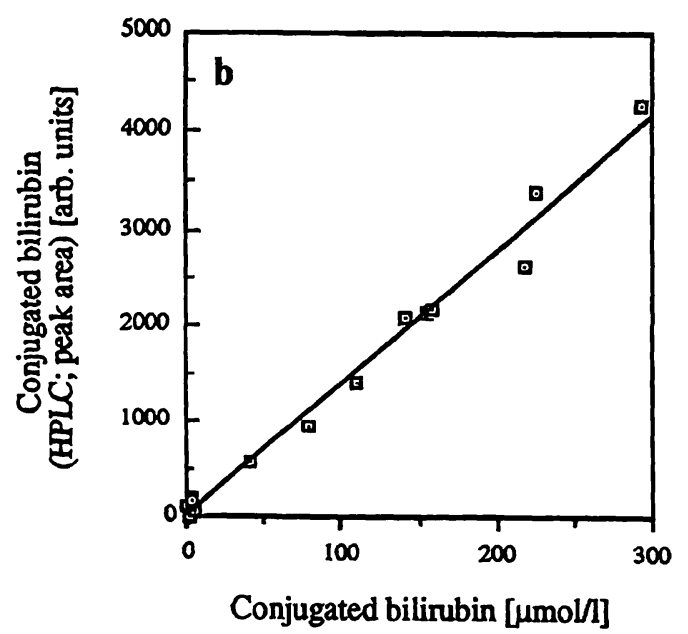

Fig. 2 Calibration curves for determining concentrations of (4Z,15Z)-bilirubin (III + IX + XIII) $\alpha$ and conjugated bilirubin from their respective HPLC peak area values (in arbitrary units).

(a) $(4 Z, 15 Z)$-Bilirubin (III $+\mathrm{IX}+\mathrm{XIII}) \alpha$ versus unconjugated bilirubin (Kodak Ektachem), $n=16$,

regression line $y=48.562 x-162.805, r=0.987, p<0.0001$.

(b) Conjugated bilirubin (HPLC) versus conjugated bilirubin (Kodak Ektachem), $n=14$,

regression line $y=13.893 x+20.294, r=0.993, p<0.0001$.

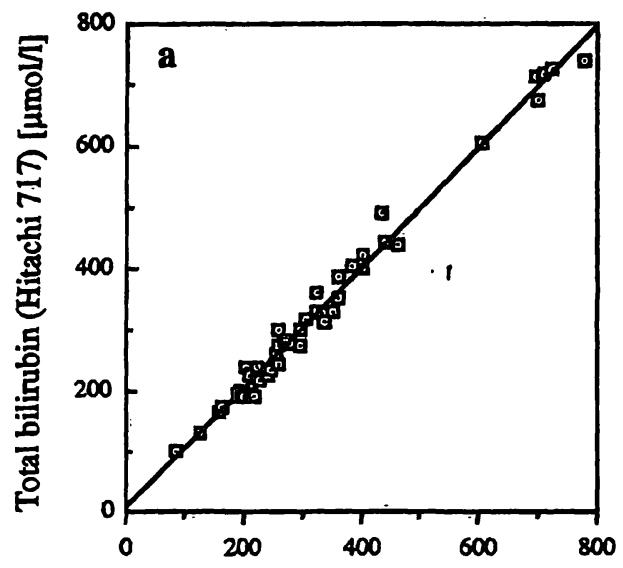

Total bilirubin (Kodak Ektachem) [ $\mu \mathrm{mol} / \mathrm{i}]$
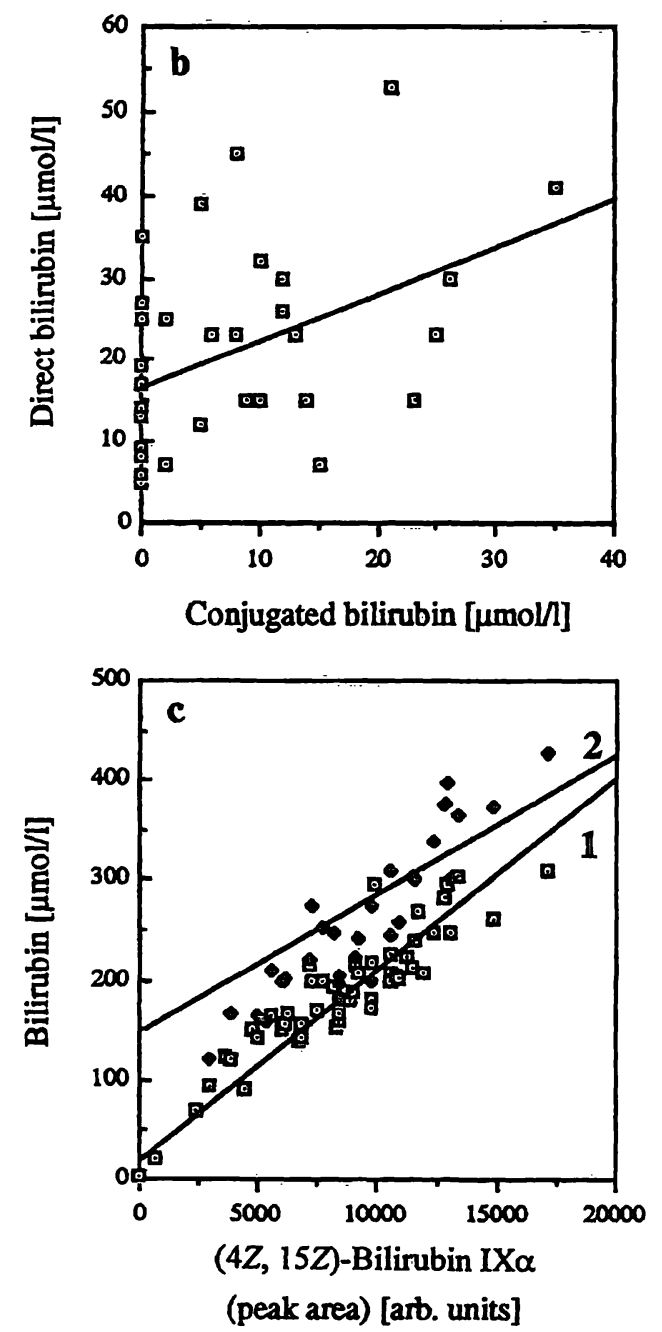

Fig. 3 Comparison of bilirubin parameters between Kodak Ektachem, Hitachi 717 and HPLC. Correlation study for:

(a) Total bilirubin (Hitachi 717) versus total bilirubin (Kodak Ektachem), $n=48$,

regression line $\mathrm{y}=0.988 \mathrm{x}+6.11, \mathrm{r}=0.993, \mathrm{p}<0.0001$.

(b) Conjugated bilirubin (Kodak Ektachem) versus direct bilirubin (Hitachi 717), $\mathrm{n}=33$,

regression line $\mathrm{y}=0.573 \mathrm{x}+16.65, \mathrm{r}=0.445, \mathrm{p}=0.0095$.

(c) (1) Unconjugated bilirubin (Kodak Ektachem) versus $(4 Z, 15 Z)-$ bilirubin (III +IX +XIII) $\alpha, \mathrm{n}=51$,

regression line $y=0.017 x+43.31, r=0.918, p<0.0001$.

(2) Indirect bilirubin (Hitachi 717 ) versus $(4 Z, 15 Z)$-bilirubin $($ III $+\mathrm{IX}+\mathrm{XIII}) \alpha, \mathrm{n}=30$,

regression line $y=0.021 x+61.82, r=0.909, p<0.0001$. 
Tab. 1 Bilirubin values from neonates determined by HPLC $(n=52)$.

\begin{tabular}{|c|c|c|c|c|c|c|}
\hline & \multicolumn{2}{|c|}{$(4 Z, 15 Z)$-Bilirubin IX $\alpha$} & \multicolumn{2}{|c|}{ Conjugated bilirubin (HPLC) } & \multirow{2}{*}{$\begin{array}{l}\text { Configurational } \\
\text { isomers } \\
\text { (peak area) }\end{array}$} & \multirow{2}{*}{$\begin{array}{l}\text { Structural } \\
\text { isomers } \\
\text { (peak area) }\end{array}$} \\
\hline & $(\text { peak area })^{\prime}$ & $(\mu \mathrm{mol} / 1)^{2}$ & $(\text { peak area })^{1}$ & $(\mu \mathrm{mol} / 1)^{3}$ & & \\
\hline Mean & 8482 & 177.0 & 127 & 7.7 & 730 & 123 \\
\hline Median & 8636 & 180.4 & 131 & 7.9 & 666 & 113 \\
\hline Range & $47-17009$ & $4.3-352.0$ & $28-220$ & $0.6-14.4$ & $78-1728$ & $15-266$ \\
\hline
\end{tabular}

' arb. units

2 calculated by means of equation (1)

3 calculated by means of equation (2)

ated with the home-made device using the appropriate filter.

\section{Bilirubin and bilirubin photoisomers}

We tested the reproducibility of preparations of native bilirubin and bilirubin photoisomers. After irradiation with blue or green light, the solution was aliquoted, frozen at $-180^{\circ} \mathrm{C}$ and stored at this temperature in the dark until further HPLC analysis. The total storage time was about 6 hours. After thawing, an aliquot was analysed at the end of each HPLC run (total time $55 \mathrm{~min}$ ). We assumed that thermal reversion of configurational isomers was negligible during analysis. Data on the reproducibility of $(4 Z, 15 Z)$-bilirubin (III + IX + XIII) $\alpha$, the sum of configurational isomers and the sum of structural isomers are summarized in table 2 . The values show that all these forms were stable, under these conditions, during the storage period.

Typical profiles of HPLC separations are given in figure 4. Figure 4a shows that under blue light, the remaining $(4 Z, 15 Z)$-bilirubin IX $\alpha$ (peak 6) and impurities such as $(4 Z, 15 Z)$-bilirubin XIII $\alpha$ (peak 5) and (4Z,15Z)-bilirubin III $\alpha$ (peak 7), are accompanied mainly by configurational isomers, especially $(4 Z, 15 E)$ )-bilirubin IX $\alpha$ (peak 3 ) and a small amount of $(4 E, 15 Z)$-bilirubin IX $\alpha$ (peak 4). Under green light (fig. $4 \mathrm{~b}$ ), the irradiation produced a large amount of structural isomers, mainly $(4 E, 15 E)$ cyclobilirubin IX $\alpha$ and $(4 E, 15 Z)$-cyclobilirubin IX $\alpha$ (peak 1 and 2 respectively); as well as an equal amount of configurational isomers (peaks 3 and 4 respectively).

Tab. 2 Reproducibility of $(4 Z, 15 Z)$-bilirubin (III + IX +XIII) $\alpha$, configurational and structural isomers $(n=7)$.

\begin{tabular}{|c|c|c|c|}
\hline & $\begin{array}{l}(4 Z, 15 Z)- \\
\text { Bilirubin } \\
\text { (III+IX } \\
+ \text { XIII) } \alpha\end{array}$ & $\begin{array}{l}\text { Config- } \\
\text { urational } \\
\text { isomers }\end{array}$ & $\begin{array}{l}\text { Structural } \\
\text { isomers }\end{array}$ \\
\hline Average peak aera* & 17856 & 2561 & 2203 \\
\hline SD & 1448 & 133 & 246 \\
\hline CV (\%) & 8.1 & 5.2 & 11.1 \\
\hline
\end{tabular}

* arb. units
At the same time, $(4 Z, 15 Z)$-bilirubin XIII $\alpha$ (peak 5) and (4Z,15Z)-bilinubin IX $\alpha$ (peak 6) are decreased.

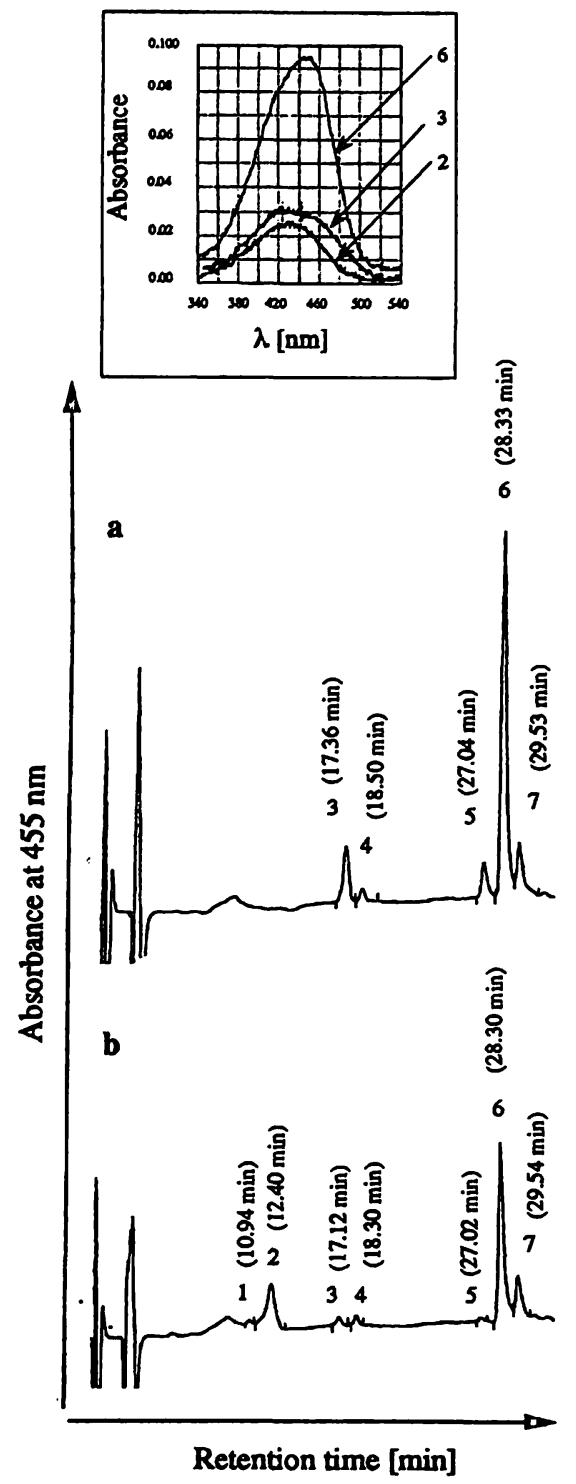

Fig. 4 Representative HPLC chromatograms of bilirubin solutions after (a) blue and (b) green light irradiation. The retention times are indicated on each peak. Peak numbering:

$1=(4 E, 15 E)$-cyclobilirubin IX $\alpha$,

$2=(4 E, 15 Z)$-cyclobilirubin $\mathrm{IX} \alpha$,

$3=(4 Z, 15 E)$-bilirubin IX $\alpha$,

$4=(4 E, 15 Z)$-bilirubin $I X \alpha$,

$5=(4 Z, 15 Z)$-bilirubin XIII $\alpha$,

$6=(4 Z, 15 Z)$-bilirubin IX $\alpha$,

$7=(4 Z, 15 Z)$-bilirubin III $\alpha$.

The insert shows absorption spectra of peaks 2, 3 and 6 in HPLC solvent. Experimental details are given in materials and methods. 


\section{Kinetics of in vitro blue and green light irradiation}

The transformation of (4Z;15Z)-bilirubin (III+ IX + XIII $) \alpha$ was followed with time. At each chosen time point of the kinetic study, the solution in the cuvette was stirred, and a sample was taken and kept at $-180^{\circ} \mathrm{C}$ in liquid nitrogen until analysis. The results are presented in figure 5. Figure 5a (blue light irradiation) shows a nearly steady-state reaction:

(i) the total peak area slowly declines and remains constant after $90 \mathrm{~s}$ at approximatively $83 \%$ of the initial total peak area;

(ii) (4Z,15Z)-bilirubin (III+IX + XIII) $\alpha$ decreases and reaches a plateau at about $90 \mathrm{~s}$ (nearly $73 \%$ of the remaining total peak area);

(iii) in the same time, the configurational isomers increase to a maximal value (nearly $28 \%$ of the remaining total peak area) and almost no changes in peak areas were detectable until $240 \mathrm{~s}$;
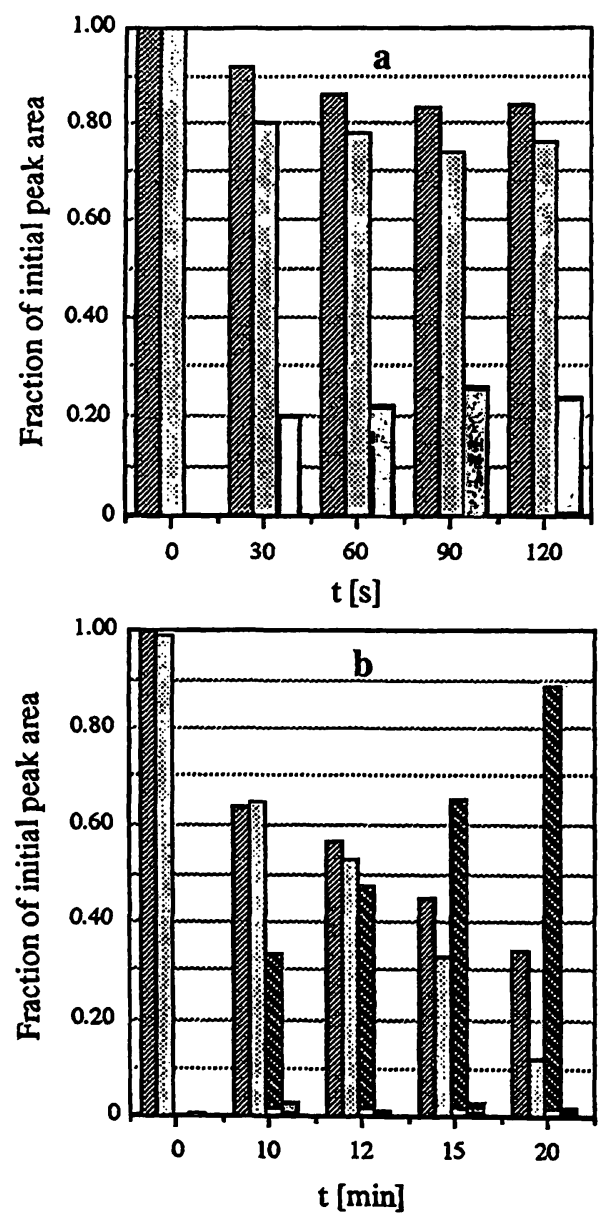

Fig. 5 Typical time course of the action of light on $(4 Z, 15 Z)$ bilirubin (III+IX+XIII) a:

(a) irradiation in blue light;

(b) irradiation in green light.

Remaining total peak area as fraction of the initial peak area.

The others fractions: $\mathbb{R}(4 Z, 15 Z)$-bilirubin (III+IX +XIII) $\alpha, \square$ configurational isomers and w structural isomers are expressed as fraction of the remaining total peak area. (iiii) during the duration of the kinetic study, no structural isomers were detected in the irradiated samples.

The kinetic data of the green photoirradiation are presented in figure $5 \mathrm{~b}$. The following changes were observed:

(i) the total peak area declines dramatically up to $33 \%$ of the initial total peak area;

(ii) in parallel, $(4 Z, 15 Z)$-bilirubin (III $+\mathrm{IX}+\mathrm{XIII}) \alpha$ disappears dramatically to $12 \%$ of the remaining total peak area;

(iii) the peak area of the structural isomers progressively increases up to a value of $88 \%$ of the remaining total peak area;

(iii) during the irradiation, the configurational isomers increase slightly but never attain a value of $2.5 \%$ of the remaining total peak area.

\section{Interference by photoisomers in routine methods for bilirubin determinations}

\section{Blue light irradiation}

The determination of the different bilirubin species, using HPLC, Kodak and Hitachi, after blue light irradiation, is presented in table 3 . These data show that:

(i) the values of total bilirubin measured by the Kodak and the Hitachi procedure are constant;

(ii) HPLC and Kodak do not detect conjugated bilirubin in samples, but the Hitachi analyser gives a constant value for direct bilirubin, and consequently also for indirect bilirubin;

(iii) the peak area of $(4 Z, 15 Z)$-bilirubin (III + IX + XIII) $\alpha$ is correlated to unconjugated bilirubin, but the equation of the regression line $(y=0.006 x+154.12, r=0.855$, $\mathrm{p}=0.0069$ ) is different from equation (1); the calculated values of unconjugated bilirubin using equation (1), are different from the measured values of unconjugated bilirubin; the differences between measured and calculated unconjugated bilirubin are correlated to the peak areas of configurational isomers $(y=0.025 x$ $+3.02, \mathrm{r}=0.858, \mathrm{p}=0.0064)$;

(iii) total bilirubin values measured by the Kodak procedure are higher than unconjugated bilirubin in spite of the absence of conjugated bilirubin in samples; by applying the appropriate formulae, the Kodak results gave a calculated value for delta bilirubin $(\delta \mathrm{B})$. These values of delta bilirubin are slightly correlated to the total peak area of configurational isomers $(y=0.006 x+14.22$, $r=0.513, p=0.194$ ). 


\section{Green light irradiation}

A representative set of data, obtained from the 3 methods after green light irradiation, is presented in table 4 . The following observations can be made:

(i) as for samples from neonates (fig. 2a), the values of total bilirubin determined on the Kodak and Hitachi were highly correlated;

(ii) surprisingly Kodak gives a false positive response for conjugated bilirubin, and conjugated bilirubin is correlated with the recorded area of structural isomers $(y=0.023 x+5.40, r=0.905, p=0.0003)$; moreover direct bilirubin values on the Hitachi are constant; (iii) unconjugated bilirubin is higher than total bilirubin measured with the Kodak procedure $\left(\mathrm{TB}_{\mathrm{Ko}}\right.$ ) (except for the lowest value of structural isomers) and also higher than the values calculated from the corresponding $(4 Z, 15 Z$ )-bilirubin (III+IX + XIII) $\alpha$ areas by means of equation (1); besides, the differences between unconjugated bilirubin and unconjugated bilirubin calculated display a reliable correlation with the areas of structural isomers $(\mathrm{y}=0.053 \mathrm{x}+14.25, \mathrm{r}=0.916, \mathrm{p}=0.0002)$;

(iiii) the peak area of $(4 Z, 15 Z)$-bilinubin (III + IX $+\mathrm{XIII}) \alpha$ is correlated with the following quantities: total bilirubin ${ }_{\text {Kodak, }}$ total bilirubin ${ }_{\text {Hitachi, }}$ indirect bilirubin and unconjugated bilirubin (fig. 6). Comparison

Tab. 3 Determination of bilirubin values after blue light irradiation?

\begin{tabular}{|c|c|c|c|c|c|c|c|c|c|c|c|}
\hline & $\begin{array}{l}\text { Configurational } \\
\text { isomers } \\
\text { (peak area) }\end{array}$ & $\begin{array}{l}(4 Z, 15 Z)-\text { Bilirubin } \\
(I I I+I X+X I I I) \alpha \\
(\text { peak area) })^{2}\end{array}$ & $\mathrm{~TB}_{\mathrm{K}_{\mathbf{0}}}$ & CB & UB & $\delta \mathrm{B}$ & $\begin{array}{l}\text { UB } \\
\text { calc }\end{array}$ & $\begin{array}{l}\text { (UB- } \\
\text { UB } \\
\text { calc) }\end{array}$ & $\mathrm{TB}_{\mathrm{Hi}}$ & DB & indB \\
\hline & $\begin{array}{r}350 \\
527 \\
487 \\
474 \\
764 \\
1110 \\
1048 \\
1067\end{array}$ & $\begin{array}{r}10204 \\
9789 \\
9377 \\
9130 \\
9095 \\
8752 \\
8615 \\
8316\end{array}$ & $\begin{array}{l}233 \\
231 \\
233 \\
234 \\
232 \\
232 \\
228 \\
228\end{array}$ & $\begin{array}{l}0 \\
0 \\
0 \\
0 \\
0 \\
0 \\
0 \\
0\end{array}$ & $\begin{array}{l}219 \\
217 \\
216 \\
214 \\
208 \\
214 \\
209 \\
206\end{array}$ & $\begin{array}{l}14 \\
14 \\
17 \\
20 \\
24 \\
18 \\
19 \\
22\end{array}$ & $\begin{array}{l}213 \\
204 \\
196 \\
191 \\
190 \\
183 \\
180 \\
174\end{array}$ & $\begin{array}{r}6 \\
13 \\
20 \\
23 \\
18 \\
31 \\
29 \\
32\end{array}$ & $\begin{array}{l}230 \\
231 \\
235 \\
233 \\
230 \\
226 \\
229 \\
226\end{array}$ & $\begin{array}{l}11 \\
11 \\
11 \\
11 \\
12 \\
11 \\
12 \\
12\end{array}$ & $\begin{array}{l}219 \\
220 \\
224 \\
222 \\
218 \\
215 \\
217 \\
214\end{array}$ \\
\hline $\begin{array}{l}\text { Mean } \\
\text { SD } \\
\text { CV (\%) }\end{array}$ & & & $\begin{array}{r}231.4 \\
2.3 \\
0.9\end{array}$ & & & & & & $\begin{array}{r}230 \\
3.1 \\
1.5\end{array}$ & $\begin{array}{r}11.4 \\
0.5 \\
4.5\end{array}$ & $\begin{array}{r}218.6 \\
3.4 \\
1.5\end{array}$ \\
\hline \multicolumn{5}{|c|}{$\begin{array}{l}1 \text { increasing amounts of configurational isomers were obtained by } \\
\text { suitable addition of irradiated sample to a non-irradiated sample } \\
\text { of bilirubin } \\
2 \text { arb. units. The other analytes are expressed in } \mu \mathrm{mol} / 1 \\
\text { Abbreviation: } \\
\mathrm{TB}_{\mathrm{Ko}}=\text { Total bilirubin, Kodak procedure } \\
\mathrm{CB} \quad=\text { Conjugated bilirubin } \\
\mathrm{UB} \quad=\text { Unconiugated bilirubin }\end{array}$} & $\begin{array}{l}\delta B \\
\mathrm{UB} \text { calc } \\
\text { UB-UB calc } \\
\mathrm{TB}_{\mathrm{Hi}} \\
\mathrm{DB} \\
\text { indB }\end{array}$ & \multicolumn{6}{|c|}{$\begin{array}{l}=\text { delta Bilirubin } \\
=\text { Unconjugated bilirubin calculated } \\
\text { equation (1) } \\
=\text { Difference } \\
=\text { Total bilirubin, Hitachi procedure } \\
=\text { Direct bilirubin } \\
=\text { Indirect bilirubin }\end{array}$} \\
\hline
\end{tabular}

Tab. 4 Determination of bilinubin values after green light irradiation ${ }^{1}$.

\begin{tabular}{|c|c|c|c|c|c|c|c|c|c|c|}
\hline & $\begin{array}{l}\text { Structural } \\
\text { isomers } \\
\text { (peak area) }\end{array}$ & $\begin{array}{l}(4 Z, 15 Z)-\text { Bilirubin } \\
(\mathrm{III}+\mathrm{IX}+\mathrm{XIII}) \alpha \\
(\text { peak area) }\end{array}$ & $\mathrm{TB}_{\mathrm{Ko}}$ & $\mathrm{CB}$ & UB & $\begin{array}{l}\text { UB } \\
\text { calc }\end{array}$ & $\begin{array}{l}\text { (UB- } \\
\text { UB } \\
\text { calc) }\end{array}$ & $\mathrm{TB}_{\mathrm{Hi}}$ & $\mathrm{DB}$ & indB \\
\hline & 184 & 8733 & 192 & 3 & 188 & 182 & 6 & 184 & 15 & 169 \\
\hline & 204 & 7198 & 164 & 13 & 181 & 151 & 30 & 167 & 14 & 153 \\
\hline & 244 & 7183 & 169 & 9 & 178 & 151 & 27 & 164 & 16 & 148 \\
\hline & 279 & 6571 & 151 & 16 & 173 & 138 & 35 & 153 & 15 & 138 \\
\hline & 357 & 5790 & 139 & 20 & 166 & 122 & 44 & 142 & 16 & 126 \\
\hline & 593 & 5473 & 144 & 16 & 165 & 116 & 49 & 140 & 17 & 123 \\
\hline & 879 & 4983 & 128 & 21 & 158 & 106 & 52 & 126 & 18 & 108 \\
\hline & 967 & 4153 & 108 & 29 & 151 & 88 & 63 & 111 & 18 & 93 \\
\hline & 1091 & 3612 & 97 & 30 & 143 & 77 & 66 & 95 & 20 & 75 \\
\hline & 1124 & 2578 & 84 & 35 & 139 & 56 & 83 & 85 & 18 & 67 \\
\hline Mean & & & & & & & & & 16.7 & \\
\hline & & & & & & & & & 1.8 & \\
\hline CV (\%) & & & & & & & & & 10.9 & \\
\hline
\end{tabular}

${ }^{1}$ increasing amounts of structural isomers were obtained by suit- $\quad{ }^{2}$ arb. units. The other analytes are expressed in $\mu \mathrm{mol} / 1$ able addition of irradiated sample to a non-irradiated sample of bili- For abbreviations see legend to tab. 3 rubin 


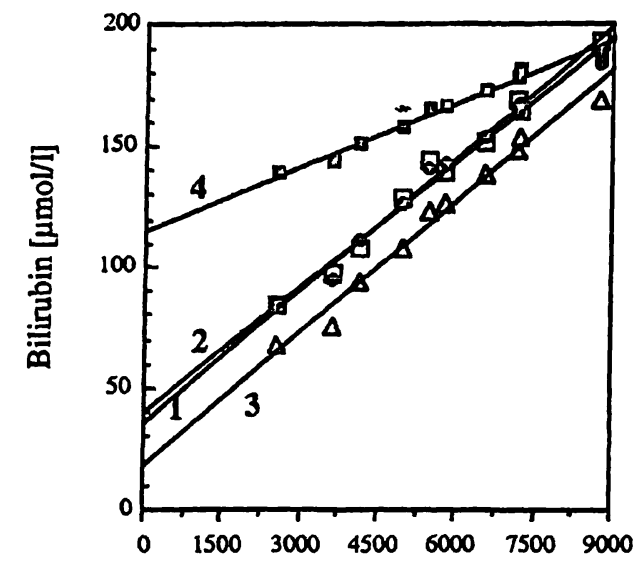

$(4 Z, 15 Z)$-Bilirubin IX $\alpha$

(peak area) [arb. units]

Fig. 6 Linear regression statistics for the following analytes : total bilirubin (Kodak Ektachem and Hitachi 717), unconjugated bilirubin (Kodak Ektachem) and indirect bilirubin (Hitachi 717) versus $(4 Z, 15 Z)$-bilirubin (III + IX + XIII) $\alpha$ determined by HPLC.

() Total bilirubin (Kodak Ektachem) versus $(4 Z, 15 Z)$-bilirubin $($ III + IX + XIII $) a, \mathrm{n}=10$,

regression line (1), $y^{\prime}=0.018 x+36.19, r=0.993, p<0.0001$. ( $\bullet$ ) Total bilirubin (Hitachi 717 ) versus $(4 Z, 15 Z)$-bilinubin $(\mathrm{III}+\mathrm{IX}+\mathrm{XIII}) \alpha, \mathrm{n}=10$,

regression line (2), $y=0.017 x+40.14, r=0.992, p<0.0001$. $(\Delta)$ Indirect bilirubin (Hitachi 717$)$ versus $(4 Z, 15 Z)$-bilirubin $(\mathrm{III}+\mathrm{LX}+\mathrm{XIII}) \alpha, \mathrm{n}=10$,

regression line (3), $y=0.018 x+18.83, r=0.991, p<0.0001$. (घ) Unconjugated bilirubin (Kodak Ektachem) versus $(4 Z, 15 Z)-$ bilirubin $(\mathrm{III}+\mathrm{IX}+\mathrm{XIII}) \alpha, \mathrm{n}=10$,

regression line (4), $y=0.009 x+115.26, r=0.991, p<0.0001$.

of these regression lines shows that total bilirubin ${ }_{\text {Kodak }}$ and total bilirubin ${ }_{\text {Hitachi }}$ are identical; the one representing indirect bilirubin is parallel to these previous 2 lines and the last unconjugated bilirubin presents a half value of slope. The intercept between unconjugated bilirubin and both total bilirubin Kodak $_{\text {and }}$ atal bilirubin Hitachi, at $_{\text {, }}$ approximately $188 \mu \mathrm{mol} / 1$ (sample without structural isomers), demonstrates that if no one of the 2 structural isomers is detected by the diazo method, these isomers give a positive response for the BUBC slide on the Kodak apparatus.

\section{Discussion}

Accurate determination of serum bilirubin fractions is needed for clinicians to survey hyperbilirubinaemic neonates. However, results may vary from laboratory to laboratory, and also in the same laboratory, depending on the methodologies and types of clinical instruments used. In addition to bias due to differences in methodologies (see for review 1.c. $(31-33)$ ), another cause of variability is the existence of photoisomers $(32,34)$. These molecular species may appear during therapeutic use of light or when samples are collected and transported without protection from light. In the first case, depending on the nature of the lamps employed, configurational isomers and structural isomers are produced; in the second case, generally only configurational isomers are produced. The specific production in quarititative amounts of these isomers, under blue and green irradiation, enabled us to demonstrate that the interference by these two kinds of isomers is not identical for the Kodak and Hitachi Analysers. In the presence of configurational isomers, the values on the Hitachi are constant (tab. 3). In spite of the variations of both $(4 Z, 15 Z)$-bilirubin (III $+\mathrm{IX}+\mathrm{XIII}) \alpha$ and configurational isomers, total bilirubin $_{\text {Hitachi }}$ quantitates all present species. The invariable direct bilirubin value in the direct methodology without caffeine and sodium benzoate reagents - is not correlated to the presence of configurational isomers. The specificity of the direct methodology is therefore deficient. In the presence of configurational isomers, the total bilirubin slide on the Kodak quantitates all present species, but the measured unconjugated bilirubin values are higher than the expected unconjugated bilirubin (UB calc). Therefore the BUBC slide is able to test configurational isomers but cannot distinguish them from the native form. The absorption spectra of $(4 Z, 15 E)$-bilirubin IX $\alpha$, the major form of configurational isomers, is slightly different from (4Z,15Z)-bilirubin IX $\alpha$ (fig. 4), reflecting the $Z \rightarrow E$ change on bond 15 of the molecule. The reflexion density enhanced to higher wavelength by the mordant in the reaction layer of the slide $(28,35$, 36) is therefore possibly different for the two species. As the differences (unconjugated bilirubin minus unconjugated bilirubin calculated (UB - UB calc) are positive, we deduce that configurational isomers are not taken totally into account by the photodetection system and the computer on the Kodak (see the correlation between (UB - UB calc) versus configurational isomers).

It has been previously reported that structural isomers were not detected by the diazo method (10), so it is not surprising that total bilirubin values on the Hitachi follow the decrease of $(4 Z, 15 Z)$-bilirubin (III + IX +XIII) $\alpha$ (tab. 4). On the other hand, the direct bilirubin values are constant. The total bilirubin slide on the Kodak based on the diazo reaction - gives the same result as the Hitachi. The conjugated bilirubin-positive results with the BUBC slide may be explained by the following hypothesis: the absorption spectra of $(4 E, 15 Z)$-cyclobilirubin IX $\alpha$, the major form of structural isomers, is shifted to shorter wavelength (maximum at around 430 $\mathrm{nm}$ ) in comparison with the native form (fig. 4); in the reaction layer, the interaction of conjugated forms with the mordant induces a shift to shorter wavelength with a maximum at $423 \mathrm{~nm}(28,35,36)$; thus, by reading the slide at $460 \mathrm{~nm}$ and particularly at $400 \mathrm{~nm}$, the solving of the computerized equations may be erroneous. Evidently, these explanations do not take account of any 
modification of the spectra of structural isomers by the mordant. This hypothesis is supported by the fact that conjugated bilirubin is highly correlated to structural isomers, but these isomers certainly contribute in the unconjugated bilirubin determination of because unconjugated bilirubin is higher than unconjugated bilirubin calculated. The extrapolation of our results to clinical situations needs caution. The excretion of photoderivatives in urine (mainly as cyclobilirubins) is markedly increased with intensive blue-light phototherapy (37). Despite their rapid excretion (38), our results show, in $38.8 \%$ of cases, that cyclobilirubins can be detected in plasma (tab. 1). Our data may explain the anomalous results reported by some authors. For example, Rosenthal \& Jennings (39) report that in samples with high unconjugated bilirubin levels the Kodak calculates delta bilirubin $(\delta B)$ values, whereas a specific manual method never detects this form; this might be explained by the presence of configurational isomers in samples (see our

\section{References}

1. Caldera R, Maynier M, Sender A, Brossard Y, Tortrat D, Galiay JC, et al. Etude de l'action de l'albumine humaine sur l'ictère en association avec la photothérapie intensive. Arch Fr Pediatr 1993; 50:399-402.

2. McDonagh AF, Agati G, Fusi F, Pratesi R. Quantum yields for laser photocyclization of bilirubin in the presence of human serum albumin. Dependence of quantum yield on excitation wavelength. Photochem Photobiol 1989; 50:305-19.

3. Landen GL, Park Y-T, Lightner DA. On the role of singlet oxygen in the self-sensitized photo-oxygenation of bilirubin and its pyrromethenone models. Tetrahedron 1983; 39:1893907.

4. Knobloch E, Hodr R, Herzmann J, Houdkova V. Kinetics of the formation of biliverdin during the photochemical oxidation of bilirubin monitored by column liquid chromatography. J Chromatogr 1986; 375:245-53.

5. Knobloch E, Mandys F, Hodr R, Hujer R, Mader R. Study of the mechanism of the photoisomerization and photooxidation of bilirubin using a model for the phototherapy of hyperbilirubinemia. J Chromatogr 1991; 566:89-99.

6. Lightner DA, Linnane III WP, Ahlfors CE. Bilirubin photooxidation products in the urine of jaundiced neonates receiving phototherapy. Pediatr Res 1984; 18:696-700.

7. Ennever JF. Blue light, green light, white light, more light: treatment of neonatal jaundice. Clin Perinatol 1990; 17:46781.

8. Ennever JF, Knox I, Speck WT. Differences in bilirubin isomer composition in infants treated with green and white light phototherapy. J Pediatr 1986; 109:119-22.

9. Itoh S, Onishi S, Isobe $K$, Manabe M, Yamakawa T. Wavelength dependence of the geometric and structural photoisomerization of bilirubin bound to human serum albumin. Biol Neonate 1987; 51:10-7.

10. Onishi $\mathrm{S}$, Itoh $\mathrm{S}$, Isobe $\mathrm{K}$. Wavelength-dependence of the relative rate constants for the main geometric and structural photoisomerisation of bilirubin IX $\alpha$ bound to human serum albumin. Biochem J 1986; 236:23-9.

11. Costarino AT, Ennever JF, Baumgart S, Speck WT, Paul M, et al. Bilirubin photoisomerization in premature neonates under low- and high-dose phototherapy. Pediatrics 1985; 75:519-22. results tab. 3). In another example, Ihara et al. (17) reported that after irradiation (between 340 and $700 \mathrm{~nm}$ ) of samples initially without conjugated bilirubin, unconjugated bilirubin is higher than total bilirubin, while conjugated bilirubin becomes positive on the Kodak; their protocol for isolating photoproducts in the aqueous phase strongly suggests the presence of a structural isomer. This result may be compared with our data presented in table 4 . The present study highlights the contribution of photoisomers in the determination of different bilirubin species. Further studies are required to explain exactly how configurational and structural isomers contribute in the quantification of bilirubin in routine clinical methodologies.

\section{Acknowledgements}

The authors wish thank Mr. G. Everett for linguistic corrections and Mrs. V. Azria for typing this manuscript.

12. Romagnoli C, De Carolis MP, Marrocco G, Tortorolo G. Failure of green light for phototherapy in preterm babies [abstract] Biol Neonate $1985 ; 43: 368-9$.

13. Tan KL. Efficacy of fluorescent daylight, blue, and green lamps in the management of nonhemolytic hyperbilirubinemia. J Pediatr 1989; 114:132-7.

14. Vecchi C, Donzelli GP, Sbrana G, Pratesi R. Phototherapy for neonatal jaundice: clinical equivalence of fluorescent green and "special" blue lamps. J Pediatr 1986; 108:452-6.

15. Ennever JF, McDonagh AF, Speck WT. Phototherapy for neonatal jaundice: optimal wavelengths of light. J Pediatr 1983; 103:295-9.

16. Ayyash $H$, Hadjigeorgiou E, Sofatzis J, Chatziioannou A, Nicolopoulos D, Sideris E. Green light phototherapy in newborn infants with $\mathrm{ABO}$ hemolytic disease. J Pediatr 1987; 111:882-7.

17. Ihara $H$, Nakamura $H$, Aoki $Y$, Aoki T, Yoshida M. In vitro effects of light on serum bilirubin subfractions measured by high-performance liquid chromatography: comparison with four routine methods. Clin Chem 1992; 38:2124-9.

18. Blijenberg BG, Roetering HA, Leijnse B. Reflections on the standardization of total bilirubin in neonatal serum. $\mathrm{J}$ Clin Chem Clin Biochem 1987; 25:177-81.

19. Mair B, Klempner LB. Abnormally high values for direct bilirubin in the serum of newborns as measured with the DuPont aca ${ }^{\circledR}$. Am J Clin Pathol 1987; 87:642-4.

20. Doumas BT, Perry BW, Jendrzejczak B, Katona V. Pitfalls in the American monitor kit methods for determination of total and "direct" bilirubin. Clin Chem 1982; 28:2305-8.

21. Ou C-N, Buffone GJ, Herr-Calomeni PJ, Finegold MJ, Shirey TL. Conjugated bilirubin versus direct bilirubin in neonates. Am J Clin Pathol 1986; 85:613-6.

22. Newman TB, Hope S, Stevenson DK. Direct bilirubin measurements in jaundiced term newborns. A reevaluation. Am J Dis Child 1991; 145:1305-9.

23. Langbaum ME, Farber SJ, Rosenthal P. Automated total and neonatal bilirubin values in newborns: is a distinction clinically relevant? Clin Chem 1992; 38:1690-3.

24. Fricker R, Brigandi T, Taylor D, Smith J, Bogovich M, Li C, et al. Analysis of total bilirubin on neonatal samples by different reagent systems [abstract]. Clin Chem 1991; 37:989. 
25. Jacobsen J. Binding of bilirubin to human serum albumin determination of the dissociation constants. FEBS Lett 1969; 5:112-4.

26. Wahlefeld AW, Herz G, Bernt E. Modification of the MalloyEvelyn method for a simple determination of total bilirubin in serum. [abstract 11.12]. Scand J Clin Lab Invest 1972; 126 Suppl:29.

27. Kodak Ektachem Test Methodology: TBil test methodology supplement recommendations for bilirubin determinations. Health Sciences Markets Division Publication MP2-39A. 1986: Rochester, New York: Eastman Kodak Compagny.

28. Wu TW, Dappen GM, Powers DM, Lo DH, Rand RN, Spayd RW. The Kodak Ektachem ${ }^{\circledR}$ clinical chemistry slide for measurement of bilirubin in newborns: principles and performance. Clin Chem 1982; 28:2366-72.

29. Knox I, Ennever JF, Speck WT. Urinary excretion of an isomer of bilirubin during phototherapy. Pediatr Res 1985; 19:198201.

30. Malhotra V, Ennever JF. Determination of the relative detector response for unstable bilirubin photoproducts without isolation. J Chromatogr 1986; 383:153-7.

31. Valaes TN, Harvey-Wilkes K. Pharmacologic approaches to the prevention and treatment of neonatal hyperbilirubinemia. Clin Perinatol 1990; 17:245-73.

32. Westwood $A$. The analysis of bilirubin in serum. Ann Clin Biochem 1991; 28:119-30.
33. Lott JA, Doumas BT. "Direct" and total bilirubin tests: contemporary problems. Clin Chem 1993; 39:641-7.

34. Sykes E, Epstein E. Laboratory measurement of bilirubin. Clin Perinatol 1990; 17:397-416.

35. Wu TW. Analyses of bilirubin fractions by HPLC and Kodak Ektachem multilayered slides. AACC National Meeting; 1985 Jul 23; Atlanta, Georgia.

36. Spayd RW, Dappen GM, Wu TW, Lauff JJ, Sundberg MW. Bilirubin-its components in serum and the Kodak assay. AACC Annual Meeting; 1982 Aug ; 8-13; Anaheim, California.

37. Ullich D, Fevery J, Sieg A, Tischler T, Bircher J. The influence of gestational age on bilirubin conjugation in newborns. Eur J Clin Invest 1991; 21:83-9.

38. Ennever JF, Costarino AT, Polin RA, Speck WT. Rapid clearance of a structural isomer of bilirubin during phototherapy. $J$ Clin Invest 1987; 79:1674-8.

39. Rosenthal $P$, Jennings MT. Serum delta bilirubin estimation by an automated method. Eur J Clin Chem Clin Biochem 1992; $30: 39-41$.

J-M. Gulian

Laboratoire de Chimie Biologique

Faculté de Médecine

27 Bd Jean Moulin

F-13385 Marseille Cedex 5

France 